\title{
A contribuição de Robert Yerkes para a engenharia humana como instrumento de dominação de classe: uma primeira aproximação
}

\section{Robert Yerkes' contribution to human engineering as an instrument of class domination: a first approximation}

\section{La contribuición de Robert Mearns Yerkes para la ingeniería humana como instrumento de dominación de clase: una primera aproximación}

\author{
André Vieira dos Santos* \\ Universidade Federal de Goiás - UFG, Goiânia, Goiás, Brasil
}

\begin{abstract}
RESUMO
O período de 1870 a 1920 é conhecido como aquele no qual diversos teóricos da psicologia estadunidense estabelecem as diretrizes teóricas e políticas da independência da disciplina frente às demais ciências e às reflexões filosóficas. A psicologia comparada proposta por Robert Mearns Yerkes é uma das mais importantes do final do século XIX e das duas primeiras décadas do século seguinte. As obras do autor referentes à evolução do sistema nervoso central e periférico e suas relações com a inteligência, em conjunto com a psicologia militar e a eugenia, possibilitam, ao menos em parte, a concretização da engenharia humana e de suas futuras aplicações em diversos setores da sociedade estadunidense como instrumento de dominação da classe dominante. Como se trata de uma obra vasta e variadas aplicações na vida cotidiana, pensamos que essa primeira aproximação seja aprofundada em futuras investigações acerca dos movimentos da classe trabalhadora durante o processo de modernização da indústria e da grande reforma social pela qual a sociedade estadunidense à época. Movimentos amplamente desprezados pela historiografia da psicologia estadunidense.
\end{abstract}

Palavras-chave: História da Psicologia, engenharia humana, Psicologia Comparada, Eugenia, Psicologia Militar.

\begin{abstract}
The period from 1870 to 1920 is known as the one in which several theorists of American psychology establish the theoretical and political guidelines of the discipline's independence from other sciences and philosophical domain. The comparative psychology proposed by Robert Mearns Yerkes is one of the most important of the late nineteenth century and the first two decades of the following century. The author's works on the evolution of the central and peripheral nervous system and its relations with intelligence, taken together with military psychology and eugenics, enable, at least in part, the concretization of human engineering and its future applications in various sectors of the American society as an instrument of domination of the ruling
\end{abstract}


class. As it is a vast work and of varied applications in everyday life, we think that this first approximation will be deepened in future investigations about the movements of the working class during the process of modernization of the industry and of the great social reform by which the American society happened to the time. These movements were widely neglected by the historiography of American psychology.

Keywords: History of Psychology, human engineering, Comparative Psychology, Eugenics, Military Psychology.

\section{RESUMEN}

El período de 1870 a 1920 es conocido como aquel en el cual diversos teóricos de la psicología estadounidense establecen las directrices teóricas y políticas de la independencia de la disciplina frente a las demás ciencias y reflexiones filosóficas. La psicología comparada propuesta por Robert Mearns Yerkes es una de las más importantes del fin del siglo XIX y de las dos primeras décadas del siglo siguiente. Las obras del autor referentes a la evolución del sistema nervioso central y periférico y sus relaciones con la inteligencia, en conjunto con la psicología militar y la eugenesia, posibilitan, al menos en parte, la concreción de la ingeniería humana y de sus futuras aplicaciones en diversos sectores de la sociedad estadounidense como instrumento de dominación de la clase dominante. Como se trata de una obra vasta y de variadas aplicaciones en la vida cotidiana, esperamos que esa primera aproximación sea profundizada en futuras investigaciones acerca de los movimientos de la clase trabajadora durante el proceso de modernización de la industria y de la gran reforma social por la que la sociedad estadounidense pasaba en esa época. Estos movimientos fueron ampliamente despreciados por la historiografía de la psicología estadounidense.

Palabras clave: Historia de la Psicología, ingeniería humana, Psicología Comparada, Eugenesia, Psicología Militar.

\section{Prolegômenos da engenharia humana: a psicologia comparada, a psicologia militar e seus laços com a eugenia}

Robert Mearns Yerkes nasceu em 26 de maio de 1876 em Bucks County, Pennsylvania. Viveu uma vida longeva e produtiva em seus quase oitenta anos, falecendo em 3 de fevereiro de 1956 em New Heaven, Connecticut. Em sua juventude tinha expectativas para seguir os estudos no campo da medicina, entretanto seu caminho mudou quando recebeu uma bolsa de estudos na Universidade de Harvard. Naquela universidade cursou apenas um ano de zoologia (1898-1899) e, em seguida, rumou para graduação em psicologia (1899-1902) defendendo sua tese de doutorado acerca das reações sensoriais e da fisiologia do sistema nervoso da medusa, em 1902, orientada por Charles C. Davenport. De 1902 a 1908 foi professor de psicologia comparada e de 1908 a 1917 promovido a professor assistente permanecendo na mesma cadeira (Yerkes, 1930; Carmichael, 1957; Hilgard, 1965).

No intervalo de tempo compreendido entre 1898 e 1921, período em que se desenvolveu a noção de engenharia humana, o autor realizou 
larga produção teórica, revisões e prefácios de diversos livros sobre variados temas tanto na Harvard University, quanto no Boston Psychopatic Hospital e também na Sanitary Corps, National Army Surgeon General's Office em Washington, quando na entrada dos Estados Unidos da América (EUA) na I Guerra Mundial em 1917.

Examinando detidamente a produção teórica de Robert Yerkes desde suas primeiras publicações até o fim da I Guerra Mundial, observamos farta reflexão epistemológica e naturalista acerca da possibilidade de delimitação entre fenômenos físicos, fisiológicos e psíquicos, dos traços estruturais e funcionais da anatomia em geral e do sistema nervoso em particular, com o objetivo de comparar os graus de consciência ou inteligência entre distintas espécies (Yerkes, 1904f, 1905b, 1910a, 1911f, 1912c, 1913d, 1913g, 1914b; Yerkes \& Urban 1906f).

Nestes artigos nota-se que, de um lado, o autor se vê diante da grande dificuldade teórica e experimental de se estabelecerem comparações entre características psicológicas humanas e infrahumanas no campo da zoologia. De outro lado, o autor não aderiu às soluções predominantes do clássico problema da relação entre mente e corpo que basicamente oscilavam entre duas perspectivas teóricas: o introspeccionismo de Titchener e o behaviorismo de Watson. A tentativa de resolução de ambos os problemas numa saída teórica e experimental foi resumida pela proposta da psicologia comparada. Com este campo de pesquisa buscava-se, primordialmente, incluir o ser humano nos procedimentos de comparação, presentes na investigação da evolução dos traços conscienciais ou de inteligência dos ditos infra-humanos, entendendo que, em algum momento da história natural das espécies, germes de consciência ou de inteligência já se encontravam em animais mais antigos que o ser humano.

À primeira vista as pesquisas de Yerkes parecem apresentar temas ou objetos diferentes, visto que abarca o estudo com diversos animais e diversos atributos físicos e psíquicos. Essa aparente dispersão se desfaz quando percebe-se que seu objeto de estudo é a evolução do sistema nervoso central e periférico (Yerkes, 1900; 1902a, 1902b, 1903d, 1904a, 1904b).

Para tanto, o autor em questão elaborou um plano de pesquisa a partir do qual ele investigou o papel: no instinto (Yerkes \& Bloomfield, 1910b); a formação do hábito e dos graus de rapidez na aquisição de novos conteúdos, sua duração e a transferência de aprendizagem para novas situações em várias espécies de animais ${ }^{1}$; as diferenças individuais presentes em diversos processos básicos psicológicos e na capacidade discriminativa dos órgãos dos sentidos (receptores), por meio do emprego de variadas estimulações com intensidades distintas e sucessivos registros das respostas de tempo de reação e de estimativa de tempo, incluindo variáveis como idade e 
sexo (Yerkes, 1899, 1901a,1902c 1903b, 1903c, 1904a, 1904d, 1905d, 1906e, 1906f, 1907,1909b, 1916b; Yerkes \& Urban, 1906d; Yerkes \& Huggins, 1903a; Yerkes \& Dodson, 1908b; Yerkes \& Eisenberg, 1915a; Coburn \& Yerkes, 1915b) e, por fim, a hereditariedade dos comportamentos (Yerkes, 1911d, 1913a).

Ao longo da execução de seu programa de pesquisa Yerkes e colaboradores encontram limitações metodológicas que são tematizada se aperfeiçoadas no âmbito experimental, quais sejam, a elaboração de novas condições de testes comportamentais, a padronização de instrumentos para estimulação das modalidades sensoriais e o registro das respostas fisiológicas e comportamentais (Yerkes, 1904c, 1904e, 1905a, 1906g, 1907, 1911a, 1912a, 1912b, 1916b; Yerkes \& Morgulis, 1909c; Yerkes \& Watson, 1911e; Yerkes \& Kellog, 1914a; Yerkes \& Coburn, 1915d).

Nas duas primeiras décadas do século XX o autor elaborou, juntamente com muitos colaboradores, o método da discriminação e amadureceu-o para o método de múltiplas escolhas e soluções de problemas. Independentemente do animal investigado, Yerkes e demais pesquisadores observaram minuciosamente os comportamentos motivados por instintos, para que depois, uma vez alcançado certo período da maturidade, se pudesse investigar a progressão da formação dos hábitos por meio da capacidade discriminativa dos animais em testes diante de estímulos diferenciados como a luz, o tamanho, a forma, a distância e a cor.

No método da discriminação os animais eram submetidos a uma situação na qual eles observavam um par de estímulos separados e situados, cada um, num compartimento. O animal deveria fazer uma escolha forçada pelo estímulo "certo", onde havia a recompensa. Caso ele escolhesse o estímulo "errado", recebia um atenuado choque elétrico nas patas. Ao longo de vários ensaios, os autores observavam que os animais escolhiam mais rapidamente e com poucos "erros" o compartimento "certo". Grosso modo, no método das múltiplas escolhas, Yerkes e colaboradores estabeleceram uma condição experimental na qual os animais observavam diversos compartimentos (entre 9 e 12 a depender da espécie) e escolhiam um deles. A consequência dessa resposta poderia ser a recompensa ou a punição. Além dos já conhecidos objetivos dos estudos da formação do hábito, o autor e seus colaboradores visavam também, dado o aumento da complexidade do experimento, identificar os graus de inteligência (formas ideacionais) intraespécies e interespécies a partir da quantificação de "erros" e "acertos". O mesmo método, inclusive, deveria ser adaptado aos seres humanos "normais" e "anormais".

Yerkes e colaboradores também elaboraram o instrumento denominado point scale para a mensuração da capacidade mental de seres humanos (Yerkes \& Bridges, 1914d; Yerkes \& Anderson, 1915c; 
Yerkes, Bridges \& Hardwick,1915e; Yerkes \& Rossy, 1917e). O point scale é uma modificação do teste de inteligência Binet-Simon aproveitando grande parte de seus itens e materiais acrescidos de modificações livremente escolhidas pelos autores. A escala tem como princípio conferir graus a diversos processos básicos psicológicos, divididos da seguinte forma: coordenação motora, percepção visual, discriminação visual, discriminação cinestésica, associação, sugestionabilidade, memória, memória auditiva, memória visual, imaginação, judicação estética, judicação prática, judicação lógica, análise e comparação e ideação (Yerkes, Bridges, \& Hardwick, 1915e, p. 10-11). A escala permite a aferição da inteligência levando-se em conta as diferenças individuais, considerando a idade, o sexo, a capacidade linguística, o status social e racial.

Yerkes também se preocupou com a produção de obras que relatavam o estado da arte da pesquisa em psicologia (Yerkes 1908a) incluindo suas preocupações com a aplicabilidade do conhecimento psicológico a partir de referenciais teórico-políticos do forte movimento eugenista estadunidense (Yerkes, 1911d, 1913f, 1914b, 1916c, 1916f). Numa definição simples, a "ciência da eugenia" investiga as linhagens humanas visando o melhoramento da raça humana. Para tanto, seus adeptos examinam exaustivamente uma grande de quantidade de características anatômicas, fisiológicas e comportamentais "normais", "anormais" e "acima da média" com fins de seleção segundo critérios supostamente neutros axiologicamente.

Importante lembrar que Yerkes era um dos principais cientistas militante da causa eugenista estadunidense. O autor fez parte da seção de eugenia da American Breeders Association e cooperava com a Eugenics Record Office por meio do Comitê de pesquisa da herança de traços mentais (Laughlin, 1913, p.9). Os debates sobre os rumos da educação de seu país e da organização do trabalho nas indústrias em pleno crescimento no início do século XX figuram no pensamento de Yerkes como locais fundamentais para a aplicação das ideias eugenistas. A psicologia comparada seria aplicada no controle e no aprimoramento dos processos básicos psicológicos. No campo da educação "o professor, tal como o engenheiro, deve ser capaz de predizer com certo grau de acurácia e certeza, os efeitos prováveis de seus métodos" (Yerkes, 1911d, p.397). Na indústria, a psicologia comparada aplicada poderia auxiliar na padronização de movimentos evitando fadiga, aumentando a eficiência e, por conseguinte, a produtividade do/a trabalhador/a (Yerkes, 1911d, p. 398)

Com a entrada dos EUA na I Guerra Mundial, Robert Yerkes se destaca como um dos principais proponentes do emprego dos conhecimentos psicológicos nas forças armadas e da formação de núcleos de treinamentos de psicologia militar para a aplicação de baterias de testes psicológicos (Yoakum \& Yerkes, 1920a; Yerkes, 1921c). Juntamente com outros psicólogos elaborou os propósitos 
originais do instrumento psicológico gestado no ano de 1917 e aplicado no ano de 1918, denominado Army Alpha e Army Beta (teste para iletrados) (Yoakum \& Yerkes, 1920a, p. xii):

1. Atribuir escores de inteligência a cada soldado com bases em exames sistemáticos;

2. A designação e a seleção de homens com inteligência superior para atribuições especiais;

3. Encorajar a seleção e a recomendação de batalhões de desenvolvimento de soldados inferiores intelectualmente, incapazes de executar as atividades diárias de um treinamento militar regular;

4. A provisão de medidas de habilidades mentais que capacitem oficiais a construírem organizações com força mental uniforme ou que busque acordo entre os graus de inteligência e as especificações definidas;

5. A seleção de homens para vários tipos de deveres militares ou para tarefas especiais, como por exemplo, treinamento em escolas militares, universidades e escolas técnicas;

6. A provisão de dados para a formação de grupos de treinamentos especiais no interior do regimento ou da bateria de maneira que cada homem deva receber instruções de acordo com sua capacidade de aprendizado;

7. A descoberta e a recomendação da eliminação de homens cuja inteligência inferior impossibilite-o de executar as atividades requeridas em quaisquer setores do serviço militar.

As baterias Army Alpha e Beta eram constituídas de testes separados. A primeira formada por testes: de direção e comandos; de aritmética; de juízos práticos; de sinônimos e antônimos; de sentenças desorganizadas; de preenchimento de números no interior de uma série de números dados; de analogias; e por fim, de informações gerais. Já segunda, por testes: de solução de problemas em labirintos desenhados; de análises de cubos; de séries do tipo X$\mathrm{O}$; de identificação de dígitos e símbolos; de checagem de números, de completar figuras; e por fim, de construção geométrica (Yoakum \& Yerkes, 1920a, p. 208-294). Cada teste era organizado com itens em ordem crescente de dificuldade e de uso exclusivo das forças armadas. A bateria foi aplicada em 35 campos de treinamento militar por uma equipe dirigida por Robert Yerkes e outros colaboradores. No total, 1.726.966 homens responderam as escalas e foram escalonados em cinco graus distintos de inteligência, em ordem decrescente de "A" a "E" (Yoakum \& Yerkes, 1920a, p. 198).

O arco temporal de vinte anos (1898 a 1918) de produção teórica de Robert Yerkes mostra-nos a grande abrangência de temas não somente voltados para atividade científica, mas também para os 
problemas da vida cotidiana. Nota-se que a preocupação teórica de Yerkes acerca da inteligência e de suas diferentes e gradativas propriedades presentes em diversas espécies de animais, a partir do estudo do sistema nervoso central, é o verdadeiro objeto central de sua pesquisa. Tal objeto esteve sempre fortemente associado aos posicionamentos políticos e econômicos de Yerkes. Nesse período, o autor advogou que o avanço das ciências biológicas, médicas e psicológicas forneceriam critérios objetivos e, portanto, imparciais, para o melhoramento da "raça humana". Desta forma, a escolha do melhor método de educação, passando pela avaliação dos níveis de inteligência cujas gradações nivelavam pessoas entre "retardados mentais" e "superdotados", "normais" e "anormais", "honestos" e "delinquentes", homens e mulheres, brancos e negros e status sociais distintos deveriam advir de estudos experimentais e da elaboração de escalas psicométricas.

\section{O amadurecimento da engenharia humana como arma da classe dominante}

Podemos dizer que esse instrumental fabricado ao longo de praticamente as duas primeiras décadas do século $X X$ contou com a figura de Yerkes para sua concreção. Após o fim da I Guerra Mundial tornou-se necessário desdobrar teórica e politicamente os resultados da psicologia comparada, da eugenia e da psicologia militar. A engenharia humana, também conhecida como engenharia mental humana é esse desdobramento. Ainda que já propalada por outros autores ao longo dos primeiros vinte anos do século passado, ela encontra o momento para se desenvolver acentuadamente.

Ao final da I Guerra Mundial, todos os esforços de cientistas e engenheiros em universidades e indústrias destinados à produção de tecnologias para o combate do inimigo precisavam ser deslocados ou readequados nos tempos ditos de paz. No campo da psicologia ficava claro que a profissionalização do psicólogo e sua independência em relação a outros ramos da ciência e da filosofia dependeram, em grande parte, da capacidade de alguns psicólogos apresentarem soluções teórico-políticas mais conformes às exigências cotidianas (O'Donnel, 1985; Napoli, 1981; Yerkes, 1920b). Robert Yerkes nos quatro anos posteriores a I Guerra Mundial produziu relatórios detalhados acerca de seu trabalho nas forças armadas, reflexões sobre a psicologia comparada e sua aplicabilidade, nos termos da engenharia humana, no porvir (Yerkes, 1918, 1919b, 1919c, 1919d, 1920b, 1921c, 1922a; Yoakum \& Yerkes, 1920a).

Em linhas gerais, a engenharia humana seria a melhor solução para as modificações estruturais de ordens política e econômica pelas 
quais passavam a sociedade estadunidense. Vejamos nas palavras do próprio intelectual (Yerkes, 1919d, p. 149):

As atividades da guerra realizadas pelo Comitê de Psicologia revelaram ou criaram oportunidades cujos significados científicos e práticos não podem ser estimados. Dois anos atrás a engenharia mental era um sonho de poucos visionários. Atualmente é um ramo da tecnologia que, embora criada na guerra, será evidentemente perpetuada e auxiliada em seu desenvolvimento pela educação e pela indústria.

O próprio sucesso dos EUA na I Guerra Mundial devia ao fato de se haver organizado cada grau de inteligência em seu posto específico. A passagem a seguir ilustra bem o pensamento do autor (Yoakum \& Yerkes, 1920a, p. 7):

A grande guerra da qual nós estamos vendo emergir uma civilização em muitos aspectos novos, tem operado mudanças maravilhosas em nossos pontos de vista, nossas expectativas e demandas práticas. Tornou-se claro para alguns indivíduos relativamente cedo que, nesta luta suprema, a utilização adequada da força humana, e mais particularmente do poder da mente ou do cérebro humano, pode assegurar a vitória final. A guerra demandou de nós a velocidade de mobilização e de treinamento de uma imensa força armada suplementar, a manufatura de munições e de armamentos de guerra em quantidades próximas do inimaginável, a construção de navios, de transportes motorizados e variedades de frotas de trens em numerosas quantidades. Tudo isso deveria ser feito o mais rápido possível. Nunca antes na história da civilização o cérebro, em vez da força bruta, foi tão importante; nunca antes a adequada utilização e o lugar do poder do cérebro foram tão essenciais para o sucesso.

O resultado da I Guerra Mundial torna-se um grande exemplo para Yerkes. O novo patamar da indústria baseada na ciência e das figuras centrais do engenheiro e do cientista com mentalidade industrial (industry-mindedscientists) nos últimos cinquenta anos nos EUA, exige dos profissionais da psicologia uma função social semelhante. Doravante, o papel social dos psicólogos será o de examinar a interação dos seres humanos com as condições objetivas nas quais estão inseridos em diversos setores da vida. A atividade do psicólogo na vida prática é constantemente comparada a de um engenheiro ou a de um cientista com mentalidade industrial. Como aponta Noble (1977, pp.45-70) a profissão do engenheiro tornara-se deveras importante no período compreendido entre 1870 e 1920, visto que 
traduzia em termos científicos e transformava em tecnologias as demandas de capitalistas. A profissão de engenheiro surgiu do acicate da concorrência entre capitalistas que competiam entre si na corrida infinda da geração e da acumulação de capital. A força de trabalho do engenheiro é vendida para que sua atuação esteja centrada na produção de novas tecnologias que aumentem a produtividade da indústria. É preciso extrair os potenciais da matéria e da energia, minimizar o custo e a autonomia dos trabalhadores habilidosos. Para tanto ele desenvolverá novas tecnologias e organizará o trabalho dentro das indústrias, desenvolvendo também procedimentos sistemáticos de patentes, organizando laboratórios com pesquisas industriais e programas extensivos de treinamento técnico. Em última, é a figura do gerenciamento científico.

Embora não conhecesse todas as determinações sociais da produção do conhecimento e da tecnologia de seu tempo, Robert Yerkes estava relativamente consciente das demandas capitalistas direcionadas à universidade. Ele vive um momento no qual a ciência e a tecnologia, de forma geral, estavam subsumidas realmente ao capital, sendo a Harvard University uma das pioneiras na formação das indústrias baseadas na ciência (Noble, 1977, p.37). Dessa forma, segundo Yerkes, o papel do psicólogo deveria ser o de investigar os fatores humanos eliminando características individuais prejudiciais e adequando a expressão das diferenças individuais da inteligência à especificidade dos postos de trabalho, tudo sob o primado do melhoramento da produtividade. Entretanto, Yerkes acreditava que a engenharia humana ainda realizava seus primeiros passos e poderia complementar os esforços das demais áreas da engenharia e, por conseguinte, das indústrias (Yerkes, 1922a, p.11):

O termo indústria empregado aqui será para designar todas as ocupações produtivas que oferecem à pesquisa dois grandes grupos de fatos e fatores: os não humanos e os humanos. $O$ primeiro inclui os objetos de estudo tais como materiais brutos da indústria, seus processos, operações, fontes de energia e cuidados mecânicos. Já o segundo, inclui fatores essenciais como a constituição física, a saúde, a inteligência, o temperamento, 0 interesse, o contentamento e a vontade de cooperar. Estes são exemplos daquelas qualidades humanas, dos traços e das relações que condicionam a atividade industrial e suas realizações. Dos dois grandes grupos de fatores presentes na indústria, os não humanos ou materiais são os mais estudados e, em alguns casos, exaustivamente, para a grande vantagem da humanidade. Exemplos podem ser tomados das contribuições da química e de outros tantos ramos da engenharia. Os fatores humanos, ao contrário, têm sido negligenciados. Apenas recentemente tem-se dado atenção ao 
significado econômico de tais pesquisas. Nós estamos no limiar de uma nova era na qual a atenção e o interesse estão começando a mudar do nível material para o pessoal, mudando das coisas com as quais se trabalha em direção ao trabalhador; da maquinaria da indústria para o homem que a construiu ou a opera. Há todas as razões para acreditar que a engenharia humana irá rapidamente ter seu lugar entre as formas mais importantes do esforço prático.

As instalações, a disponibilidade da matéria-prima e a evitação de seu desperdício, o controle dos aspectos físicos e mentais do trabalhador nos processos de trabalho envolvidos na produção de mercadorias ficariam a encargo dos engenheiros dos fatores humanos e não humanos. Estes trabalhariam ajustando todos os fatos e fatores dos espaços de trabalho e da força de trabalho ao imperativo da produtividade, da geração de valor e de mais-valor para a concentração nas mãos de capitalistas.

Podemos dizer que com a concretização da indústria baseada na ciência e, portanto, da subordinação real da ciência e da tecnologia ao capitalismo nos EUA, a psicologia comparada, a psicologia militar, a eugenia e a engenharia humana foram expressões teórico-políticas de um momento histórico que se propuseram a aperfeiçoar os processos de produção de mercadorias, por meio do controle fino das capacidades humanas, a atuar como possível gestor de crises econômicas e de conflitos sociais variados, tais como as grandes greves de trabalhadores/as nas indústrias. Sabemos que os estudos de zoologia e de psicologia comparada ofereceram objetivamente dados interessantes acerca da evolução do sistema nervoso e periférico de várias espécies. Entretanto, com a acentuação da subordinação da ciência e da tecnologia ao capital a objetividade de tais estudos tornou-se comprometida.

Analisando as condições experimentais dos estudos de psicologia comparada, da aplicação do método de múltiplas escolhas a diversas espécies, bem como os itens das escalas, todos produzidos por Yerkes e colaboradores, não é difícil afirmar que tanto a redução do ambiente animal a labirintos, caixas-problema ou a salas fechadas artificiais com situações-problemas, quanto a redução das situações da vida cotidiana em itens de escalas, representavam de forma simplificada os diversos espaços dos setores da sociedade civil estadunidense, como os espaços de trabalho, de educação, da família etc. É que no pensamento de Yerkes as ferramentas teóricas darwinianas da analogia e da homologia estão imiscuídas, indissociadas às necessidades eugênicas, da psicologia militar e da engenharia humana. Os animais adentram em caixas e salas. São extremamente adequados a uma condição completamente artificial que tolhem seus movimentos, até que se tornem aptos a executar 
tarefas muito específicas, jamais ou pouco vistas na natureza. Os comportamentos executados nestas condições artificiais podem ser acompanhados do risco de punições como choque elétrico ou isolamento e recompensas como comida, descanso e/ou contato físico com coespecíficos.

É bastante conhecida a situação da classe trabalhadora nos EUA no mesmo período em que se gesta a eugenia e a engenharia humana, embora grande parte da história da psicologia daquele país a invisibilize ou a sussurre, vez por outra. Trata-se da história de extrema penúria, da coerção econômica de massas humanas que não tinham alternativas, a não ser vender sua força de trabalho para os capitalistas industriais. Nestes espaços, alheios à sua vida cotidiana e devidamente construídos para a produção de mercadorias, a condição para que ganhassem seus salários e garantissem minimamente sua subsistência era aprender rapidamente todos os movimentos necessários no processo de trabalho. Em última, ser um apêndice da máquina ou ser desligado do emprego. Uma vez amadurecida a engenharia humana, ela atuaria como atenuadora dos possíveis conflitos entre os/as trabalhadores/as visto que voltada para os fatores humanos e na melhor adequação da inteligência aos postos de trabalho, atenuando conflitos e permitindo um tipo de amoldamento mais preciso da classe trabalhadora às transformações pelas quais passavam as indústrias após a I Guerra Mundial. Os labirintos, caixas-problema ou salas de experimentos, bem como as escalas de Yerkes são um misto da impossibilidade técnica da época de se observarem os animais em ambientes mais próximos aos naturais e, ao mesmo tempo, a busca da representação simplificada de espaços como fábricas, escolas e congêneres.

Todavia, não é somente na esfera da produção de mercadorias que repousam as reflexões de Yerkes. São igualmente importantes, até sua morte, suas reflexões sobre o "problema da imigração", a delinquência, a escolha de melhores métodos de educação, o desempenho de um papel chave na coordenação de pesquisadores/as nos esforços da II Guerra Mundial, nos estudos de psicopatia e outros temas de que falaremos numa outra oportunidade.

\section{O pensamento de Robert Yerkes num contexto histórico mais amplo}

Até aqui fizemos uma primeira aproximação à obra de Robert Yerkes e de sua contribuição para o nascimento da engenharia humana. Num contexto histórico mais amplo, este estudo nos oferecerá as bases a partir das quais a engenharia humana se desenvolverá ao longo dos séculos XX e XXI. É que nosso estudo sobre o pensamento de Yerkes faz parte de uma investigação de maior envergadura que 
visa tornar inteligível como a psicologia ligada às ciências naturais, sobretudo à biologia, desenvolvem formulações teóricas, tecnologias e políticas como instrumentos para a dominação da classe trabalhadora. Ela está dividida em momentos importantes da história dos Estados Unidos desde a Guerra de Secessão e o amadurecimento do capitalismo industrial no fim do século XIX, passando pela concretização da hegemonia deste país mundialmente, sobretudo após a I Guerra Mundial, até o lançamento da iniciativa BRAIN no segundo governo Barack Obama em 2013 (Vieira, 2019).

Os anos entre 1890 e 1922 são conhecidos entre os historiadores da psicologia estadunidense como "a psicologia do recente século XX" e têm sido objeto de muitas investigações. Isto se deve ao fato de ficarem cada vez mais patente, entre os pesquisadores, as missões de criarem-se espaços dentro e fora da academia que auxiliassem tanto no surgimento da psicologia como ciência, afastando-a de reflexões metafísicas, quanto de sua nacionalização e profissionalização. Vale lembrar que é neste período que surgem a American Psychology Association (APA) em 1892 e diversos meios de comunicação dos resultados de pesquisas psicológicas, ambos imbuídos daquelas missões.

Nestes trinta e dois anos também observam-se os autores da psicologia comparada e do behaviorismo, da psicometria (elaboradores e aplicadores de testes mentais) e da psicologia estruturalista digladiando-se sobre a possibilidade da elaboração de tecnologias voltadas para as prementes questões sociais da época. A Psicologia como disciplina autônoma, sua nacionalização e sua profissionalização dependeram da resolução desta questão. Os fundadores da APA e de vários periódicos de psicologia estavam convencidos da utilidade da recém-criada disciplina. O'Donnel (1985, p. 195-200) apontou o quão problemático foi para alguns psicólogos recém doutores, principalmente oriundos da psicologia comparada, ocuparem um cargo nas universidades se não tratassem, de forma aplicada, das "questões práticas humanas". Abdicando de seus objetos de estudo, grande parte deles rumaram para universidades, ministraram aulas e elaboraram pesquisas aplicadas nos campos da psicologia da educação e da orientação vocacional, construindo e trabalhando com testes psicológicos. Havia, portanto, circunstâncias econômicas e políticas que empurravam os psicólogos em direção à prática.

Paulatinamente, se observarmos o devir desses campos, vemos que os autores da psicologia comparada, do behaviorismo e, de certa forma, alguns estruturalistas, almejavam tanto a separação da psicologia de seu passado metafísico, quanto criticavam o estabelecimento de uma "ciência pura", destacada da aplicabilidade. Já os psicólogos envolvidos com o tema da aprendizagem e da educação já primavam, em sua grande parte, desde a última década 
do século XIX, na elaboração dos conceitos psicológicos e ferramentas, tais como os testes mentais, a partir de problemas cotidianos.

As investigações de Donald S. Napoli (1981), John O'Donnel (1985) e Kurt Danziger (1990), sobre o surgimento da psicologia aplicada, as origens do behaviorismo, da psicologia comparada e do neogaltonismo nos Estados Unidos da América (EUA) mostram como elas estão intimamente ligadas ao processo profissionalização da psicologia estadunidense. O'Donnel (1985, p. 9) atribui às universidades, cada uma à sua maneira, o papel de traduzir as "pressões culturais e socioeconômicas" da sociedade estadunidense em determinadas diretrizes científicas e tecnológicas para os quadros acadêmicos. Já Napoli (1981, p. 5), chamou atenção para o surgimento da psicologia aplicada frente às "mudanças na sociedade americana" no que diz respeito à complexidade e ao tamanho de suas instituições. Entretanto, a investigação dessas "pressões" e como elas se exercem sobre as universidades ainda carecem de aprofundamento.

Estamos mais próximos de Danziger (1990, p.103) quando explica o processo de profissionalização da psicologia a partir de exigências dos contextos sociais mais amplos, oriundos do industrialismo corporativo. Em resumidas linhas, explica o autor (Danziger, 1990, p. 101):

O fato é que quase desde o início do século XX a psicologia tem cessado de ser uma disciplina acadêmica pura e iniciado a comercialização de seus produtos para o mundo. Isto significa que as condições de seu mercado potencial foram capazes de influenciar em quais direções suas práticas investigativas foram mais prováveis de se desenvolverem. Práticas que foram mais úteis na construção de produtos vendáveis específicos se tornaram mais prováveis de serem encorajadas, ao passo que outras faltosas dessa capacidade, escanteadas).

Danziger observou que o enorme crescimento das high schools foi acompanhado de demandas gerenciais dos administradores daquelas escolas direcionadas aos pesquisadores em psicologia. Em 1890, por exemplo, Granville Stanley Hall foi um dos primeiros psicólogos a estabelecer claras relações com professores desenvolvendo práticas investigativas censitárias. Com o crescimento das escolas e das alianças em termos de pesquisas e produção de tecnologias educacionais, surgiu uma nova geração de administradores educacionais, profissionais que gerenciavam as escolas a partir das ferramentas psicoeducacionais.

Nesta época as escolas eram claramente organizadas aos moldes das indústrias. Salvando as diferenças entre estes espaços era necessário 
tanto um método de ensino eficaz para ajustar o trabalhador ao uso das instalações, das máquinas e das matérias-primas, bem como de regras de convívio dentro do espaço de trabalho, quanto para as crianças e adolescentes em salas de aula. Nestes espaços, mediante o teste de vários métodos de ensino para o ajustamento, ambas as performances eram observadas, mensuradas e comparadas em condições distintas. Isso possibilitava a análise do método de ensino que assegurava melhores resultados em detrimento de outros.

Estamos de acordo com os autores acima de que existem relações entre as universidades e o todo social no qual ela se insere. Também pensamos que cada universidade tem sua particularidade ao mediar as demandas societais de uma época. No interior dessas mesmas universidades há que se considerar, os papéis sociais de seus agentes, os valores que dirigem suas ações, as expectativas dos pesquisadores frente às diretrizes científicas e tecnológicas de diversas instituições (fundações de pesquisa, indústrias e agências governamentais), quais pesquisas recebem fomento e mantêm sua vitalidade em detrimento de outras, a competitividade entre pesquisadores diante de recursos escassos e os meios para obtê-los.

Entretanto, a nosso ver, o contexto social mais amplo caracterizado como industrialismo corporativo por Danziger (1990) deixa de lado as profundas modificações políticas e econômicas ocorridas nos EUA após a Guerra de Secessão (1861-1865). Para tanto, acreditamos que num futuro próximo para entender a produção teórico-política de Robert Yerkes será preciso investigar como se constituem as relações sociais capitalistas nos EUA. Isto por um motivo simples: os pesquisadores são seres humanos e estão, necessariamente, numa divisão social do trabalho científico e tecnológico que, por sua vez, são partícipes das relações sociais de produção e de reprodução das riquezas. Mudanças nessas relações interferem naquela divisão. Por sua vez, modificações na divisão incidem sobre as relações sociais. Um dos primeiros passos que estamos vislumbrando para se entender a nascente psicologia estadunidense é capturar o movimento da sociedade civil burguesa estadunidense. Começamos por Robert Mearns Yerkes devido ao fato de ele ter sido uma personagem fundamental para o surgimento da engenharia humana, umas das expressões do processo de profissionalização da psicologia e de sua independência.

Os aspectos que julgamos relevantes nas investigações de Danziger (1990), O'Donnel (1985) e de Napoli (1981) serão integrados num estudo histórico que parte da categoria da totalidade e ajuda a compreender a história da psicologia estadunidense no seio das relações sociais capitalistas daquele país. É preciso compreender a particularidade das relações sociais capitalistas no período investigado neste artigo para que entendamos a discussão acerca das mudanças no seio da história da psicologia neste momento e, por 
conseguinte, o que nos é mais caro, o papel da psicologia estadunidense e da engenharia humana na I Guerra Mundial.

Está muito claro para nós o grave silenciamento na história da psicologia estadunidense das lutas cotidianas da classe trabalhadora. As modificações políticas e econômicas, a modernização do espaço urbano, a acentuação da industrialização, as políticas de higiene e de imigração entre outras, não existiram sem os conflitos diretos com a classe trabalhadora. Inúmeras revoltas surgiram dos chãos das fábricas contra diversas formas de gerenciamento científico (Phoner, 1965; Montgomery, 1979).

Como conclusão parcial deste escrito pensamos que a independência teórica da psicologia estadunidense de uma forma geral e a formulação da engenharia humana em particular é uma das respostas da classe dominante às lutas da classe trabalhadora por mais direitos sociais no final do século XIX e início do século XX. A função social do psicólogo e do engenheiro ligado às questões humanas é produzir uma consciência social sob a forma de ideologia, amoldando a classe trabalhadora à sociabilidade do capital (Marx \& Engels, 2007).

Além disso, com término da I Guerra Mundial os conflitos com outros países não cessam. O imperialismo estadunidense se espraia para diversos países ao longo de todo o século XX, fenômeno igualmente pouco comentado na historiografia da psicologia daquele país. Mais investigações serão necessárias acerca da vida e das obras Robert Yerkes. Elas auxiliarão no desvelamento de uma rede de psicólogos com os quais ele trabalhava, figuras igualmente responsáveis pela difusão de tal ideologia, da direção de importantes instituições científicas, agências governamentais e fundações, da formulação de diretrizes político-científicas, de valores e de missões norteadores das práticas psicológicas.

\section{Referências}

Coburn, C. A., \& Yerkes, R. M. (1915b). A study of the behavior of the crow Corvus Americanus Aud. by the multiple choice method. J ournal of Animal Behavior, 5(2), 75-114.

Carmichael, L. (1957). Robert Mearns Yerkes 1876-1956. The Psychological Review, 64(1), 1-7.

Danziger, K. (1990). Constructing the Subject: Historical Origins of Psychological Research. Cambridge: Cambridge University Press.

Hilgard, E. R. (1965). “Robert Mearns Yerkes, May 26, 1876-February 3, 1956". Biographical Memoirs National Academy of Sciences, $38,385-425$. 
Laughlin, H. Hamilton, Carnegie Institution of Washington. Eugenics Record Office. (1913). Report no. 1. Cold Spring Harbor, N.Y.: [The Office].

Marx, K., \& Engels, F. ([1845-1846] 2007). A ideologia alemã: Crítica da novíssima filosofia alemã em seus representantes Feuerbach, B. Bauer e Stirner, e do socialismo alemão em seus diferentes profetas. Rio de J aneiro: Civilização Brasileira.

Montgomery, D. (1979). Workers' control in America: Studies in history of work, technology and labor struggles. Cambridge: Cambridge Universities Press.

Napoli, D. S. (1981). Architects of adjustement. The history of the psychological profession in the United States. Port Washington, N.Y., London: National University Publications, Kennikat Press.

Noble, D. F. (1977). American by design. Science, technology and the rise of corporate capitalism. NewYork: Alfred A. Knopf.

O'Donnel, J. M. (1985). The origins of behaviorism. American Psychology, 1870-1920. New York: New York University Press.

Phoner. P. S. (1965). History of the labor movement in the United States: The industrial workers of the world, 1905-1917 (Vol. 5). New York: International Publishers.

Vieira, André S. (2019). Capital-imperialismo e psicologia experimental: A Brain Initiative como estudo de caso. Trabalho não publicado.

Yerkes, R. M. (1899). Reaction of entomostaca to stimulation by light. American J ournal of Physiology, 3(4), 157-182.

Yerkes, R. M. (1900). Reaction of Entomostraca to stimulation by light II: Reactions of Daphnia and Cypris. American Journal of Physiology, 4(8), 405-22.

Yerkes, R. M. (1901). A Study of Variation in the Fiddler Crab Gelasimus Pugilator Latr. Proceedings of the American Academy of Arts and Sciences, 36(24), p. 417-442.

Yerkes, R. M. (1902a). A Contribution to the Physiology of the Nervous System of the Medusa Gonionema Murbachi: Part I.The Physiology of the Central Nervous System. American. Journal of Physiology, 6(6), p. 434-449.

Yerkes, R. M. (1902b). A Contribution to the Physiology of the Nervous System of the Medusa Gonionema Murbachii: Part II.The Physiology of the Central Nervous System. American. Journal of Physiology, 7(2), p. 181-197.

Yerkes, R. M. (1902c). Habit-Formation in the Green Crab, Carcinus granulatus. Biological Bulletin, 3(5), 241-244.

Yerkes, R. M. \& Huggins, G. E. (1903a). Habit-Formation in the Crawfish Cambarus Affinis. Harvard Psychological Studies, 1, 565-577. 
Yerkes, R. M. (1903b). The Instincts, Habits and Reactions of the Frog. The Psychological Review: Monograph Supplements, 4(1), 579-638.

Yerkes, R. M. (1903c). A Study of the Reactions and Reaction-Time of the Medusa Gonionemamurbachii to Photic stimuli. American. Journal of Physiology, 9(5), 279-307.

Yerkes, R. M. (1903d). Reactions of Daphnia pulex to light and heat. In Mark Anniversary Volume (pp. 359-377). New York: Henry Holt Co.

Yerkes, R. M. (1904a). The reaction-time of Gonionemusmurbachii to electric and photicstimuli. Biological Bulletin, 6(2), 84-95.

Yerkes, R. M. (1904b). Space perception of tortoises. The Journal of Comparative Neurology and Psychology, 14(1), 17-26.

Yerkes, R. M. (1904c). Variability of reaction-time. Psychological Bulletin, 1(5), 137-146.

Yerkes, R. M. (1904d). Inhibition and reinforcement of reaction in the frog rana clamitans. The Journal of Comparative Neurology, 14(2), 124-137.

Yerkes, R. M. (1904e). The use of roman numerals. Science, 20(55), 309-310.

Yerkes, R. M. (1904f). Editorial: Physiology and psychology. The Journal of comparative psychology, 14(6), 511-514. doi: $10.1002 /$ cne.920140603

Yerkes, R. M. (1905a). Concerning the genetic relations of types of action. Journal of Comparative Neurology and Psychology, $15(2), 132-137$.

Yerkes, R. M. (1905b). Animal Psychology and Criteria of the Psychic. The Journal of Philosophy psychology and scientific methods, 2(6), 141-149.

Yerkes, R. M. (1905d). The sense of hearing in frogs. The Journal of Comparative Neurology and Psychology, 15(2), 279-304.

Yerkes, R. M., \& Urban, F. M. (1906d). Time-estimation in its relations to sex, age, and physiological rhythms. Harvard Psychology Studies, 2, 405-30.

Yerkes, R. M. (1906e). The mutual relations of stimuli in the frog Rana Clamata Daudin. Harvard Psychology Studies, 2, 545-574.

Yerkes, R. M., \& Urban, F. M. (1906f). The temporal relations of neural process. Harvard Psychology Studies, 2, 575-580.

Yerkes, R. M. (1906g). Concerning the behavior of Gonionemus. Journal of Comparative Neurology and Psychology, 16(6), 457463

Yerkes, R. M. (1906f). The temporal relations of neural process. Harvard Psychology Studies, 2, 575-580.

Yerkes, R. M. (1907). The dancing mouse: a study in animal behavior. New York: The Macmillan company. 
Yerkes, R. M. (1908a). Recent progress and present tendencies in comparative psychology. Journal of Abnormal and Social Psychology, 2, 271-79.

Yerkes, R. M., \& Dodson, J. D. (1908b). The relation of strength of stimulus to rapidity of habit-formation. Journal of Comparative Neurology and Psychology, 18(5), 459-482

Yerkes, R. M., \& Berry, C. S. (1909a). The Association Reaction Method of Mental Diagnosis (Tatbestandsdiagnostik). The American J ournal of Psychology, 20(1), 22-37.

Yerkes, R. M. (1909b). Modifiability of behavior in its relations to the age and sex of the dancing mouse. Journal of Comparative Neurology and Psychology, 19(3), 237-271.

Yerkes, R. M., \& Morgulis, S. (1909c). The method of Pawlow in animal psychology. Psychological Bulletin, 6(8), 257-273.

Yerkes, R. M. (1910a). Psychology and its relation to biology. Journal of philosophical psychology and scientific methods, 7(5), 113124.

Yerkes, R. M., \& Bloomfield, D. (1910b). Do kittens instinctively kill mice?. Psychological Bulletin, 7(8), 253-263.

Yerkes, R. M. (1911a). The psychological aspects of illuminating engineering: lectures on Illuminating Engineering delivered at J ohns Hopkins University. Baltimore: Johns Hopkins Press.

Yerkes, R. M. (1911d). Introduction to Psychology. New York: Nery Holt and Company.

Yerkes, R. M., \& Watson, J. B. (1911e). Methods of studying vision in animals. Behavior Monography, 1(2), 1-86. Recuperado em 13 de outubro de 2018, de https: // babel. hathitrust. org/cgi/pt?id=mdp.39015035573297; vi ew $=1$ up; seq $=101$

Yerkes, R. M. (1911f). Instinct. Psychological Bulletin, 8(11), 395397. Recuperado em 13 de outubro de 2018, de https: // babel. hathitrust. org/cgi/pt?id=hvd. hnux 5 w; view=1up; s eq $=451$

Yerkes, R. M. (1912a). The class experiment in psychology with advertisements as materials. The Journal of Educational Psychology, 3(1), 1-17.

Yerkes, R. M. (1912b). The discrimination method. Journal of Animal Behavior, 2(2), 142-44.

Yerkes, R. M. (1912c). The intelligence of earthworms. Journal of Animal Behavior, 2(5), 332-352.

Yerkes, R. M. (1913a). The heredity of savageness and wildness in rats. J ournal of Animal Behavior, 3(4), 286-296.

Yerkes, R. M. (1913d). Comparative Psychology: A Question of Definitions. Journal Philosophical Psychology and Scientific Methods, 10, 580-82. 
Yerkes, R. M. (1913f). Outline of study of the self. Cambridge: Harvard Universities.

Yerkes, R. M. (1913g). Comparative Psychology in Relation to Medicine. Boston Medical Surgical J ournal, 169(22), 779-781.

Yerkes, R. M., \& Kellog, C. E. (1914a). A graphic method of recording maze-reactions. Journal of Animal Behavior, 4(1), 50-55.

Yerkes, R. M. (1914b). The study of human behavior. Science, 39(1009), 625-633.

Yerkes, R. M., \& Bridges, J. W. (1914d). The point scale: a new method for measuring mental capacity. Boston Medical and Surgical J ournal, 171(23), 857-66.

Yerkes, R. M., \& Eisenberg, A. M. (1915a). Preliminaries to a study of color vision in the ring-dove Turturrisorius. Journal of Animal Behavior, 5(1), 25-43.

Yerkes, R. M., \& Anderson, H. M. (1915c). The importance of social status as indicated by the results of the Point-Scale method of measuring mental capacity. Journal of Educational Psychology, 6(3), 137-150.

Yerkes, R. M., \& Coburn, C. A. (1915d). A study of the behavior of the pig SusScrofa by the multiple choice method. Journal of Animal Behavior, 5(3), 185-225.

Yerkes, R. M., Bridges J. W., \& Hardwick, R. S. (1915e). A Point Scale for Measuring Mental Ability. Baltimore: Warwick and York.

Yerkes, R. M. (1916b). The mental life of monkeys and apes: a study of ideational behavior. Behavior Monographs, 3(1). doi: 10.5962/bhl. title. 56174

Yerkes, R. M. (1916c). Mental examination of police and court cases. Journal of the American Institute of Criminal Law and Criminology, 7(3), 366-72.

Yerkes, R. M. (1916f). Educational and psychological aspects of racial well-being. Journal of Delinquency, 1(5), 243-49. Recuperado em 11 de dezembro de 2018, de https: // babel. hathitrust. org/cgi/pt?id=hvd. hl1zbp; view=1up; se $q=263$

Yerkes, R. M., \& Rossy, C. S. (1917e). A point scale for the measurement of intelligence in adolescent and adult individuals. Boston Medical Surgical J ournal, 176(16), 564-573.

Yerkes, R. M. (1917). The psychological examination of recruits. The Scientific Monthly, 5(5), 478-479.

Yerkes, R. M. (1918). Psychology in relation to the war. Psychological Review, 25(2), 85-115.

Yerkes, R. M. (1919b). The measurement and utilization of brain power in the army. Science, 49(1262), 221-226.

Yerkes, R. M. (1919c). The measurement and utilization of brain power in the army: Part II. Science, 49(1263), 251-59. 
Yerkes, R. M. (1919d). Report of Psychology Committee of the National Research Council. Psychological Review, 26(2), 83149.

Yerkes, R. M. (1920b). The New World of Science: its development during the war. New York: Century Co.

Yerkes. R. M. (1921c). Psychological examining of the army in USA. Washington, D.C.: Washington Government Printing Office.

Yerkes, R. M. (1922a). What is personel research?. Monthly Labor Review, 14(1), 11-18.

Yerkes. R. M. (1930). Autobiography of Robert Mearns Yerkes. In C. Murchinson (Ed.), History of Psychology in Autobiography (Vol. 2, pp. 381-407). Worcester, Massachusetts: Clark University Press.

Yoakum, C. S., \& Yerkes, R. M. (1920a). Army Mental Tests. New York: Henry Holt and Company.

\section{Endereço para correspondência André Vieira dos Santos \\ Universidade Federal de Goiás \\ Grupo Crise - Programa de Pós-graduação em Psicologia - Faculdade de Educação Rua 235, s/n, Setor Leste Universitário, CEP 74605-050, Goiânia - GO, Brasil \\ Endereço eletrônico: andrevieira@ufg.br}

Recebido em: 19/12/2018

Reformulado em: 13/02/2019

Aceito em: 14/02/2019

\section{Notas}

* Possui graduação em Psicologia pela Universidade Federal do Rio de Janeiro (2005). É doutor pela mesma universidade (2013). Tem experiência na área de Fisiologia, com ênfase em Neurofisiologia, Fisiologia do Comportamento e Psicobiologia do Estresse, bem como nas áreas de História e Epistemologia da Psicologia. Atuou nos seguintes temas de pesquisa: Regulação da Emoção, Fatores de Vulnerabilidade ao Estresse, Psiconeuroendocrinologia, Reflexos Somáticos e Modulação dos Padrões Posturais em seres humanos. Atualmente, investiga a história da psicologia e das neurociências, suas produções conceituais, inovações tecnológicas e seus impactos sobre a sociedade contemporânea.

${ }^{1}$ Notadamente algumas espécies do subfilo crustáceo, espécies de tartarugas, de sapos, de medusas, de ratos, de minhocas, corvos, de porcos, de macacos e também estudos com seres humanos.

Financiamento: CAPES.

Este artigo de revista Estudos e Pesquisas em Psicologia é licenciado sob uma Licença Creative Commons Atribuição-Não Comercial 3.0 Não Adaptada. 